\title{
ARTICLE
}

Received 4 May 2012 | Accepted 17 Dec 2012 | Published 22 Jan 2013 DOI: 10.1038/ncomms2394

\section{A high-mobility two-dimensional electron gas at the spinel/perovskite interface of $\gamma-\mathrm{Al}_{2} \mathrm{O}_{3} / \mathrm{SrTiO}_{3}$}

Y.Z. Chen ${ }^{1}$, N. Bovet ${ }^{2}$, F. Trier ${ }^{1}$, D.V. Christensen ${ }^{1}$, F.M. Qu ${ }^{3}$, N.H. Andersen ${ }^{4}$, T. Kasama ${ }^{5}$, W. Zhang ${ }^{1}$, R. Giraud ${ }^{6,7}$, J. Dufouleur ${ }^{6}$, T.S. Jespersen ${ }^{8}$, J.R. Sun ${ }^{3}$, A. Smith', J. Nygård ${ }^{8}$, L. Lü ${ }^{3}$, B. Büchner ${ }^{6}$, B.G. Shen ${ }^{3}$, S. Linderoth ${ }^{1}$ $\&$ N. Pryds ${ }^{1}$

The discovery of two-dimensional electron gases at the heterointerface between two insulating perovskite-type oxides, such as $\mathrm{LaAlO}_{3}$ and $\mathrm{SrTiO}_{3}$, provides opportunities for a new generation of all-oxide electronic devices. Key challenges remain for achieving interfacial electron mobilities much beyond the current value of approximately $1,000 \mathrm{~cm}^{2} \mathrm{~V}^{-1} \mathrm{~s}^{-1}$ (at low temperatures). Here we create a new type of two-dimensional electron gas at the heterointerface between $\mathrm{SrTiO}_{3}$ and a spinel $\gamma-\mathrm{Al}_{2} \mathrm{O}_{3}$ epitaxial film with compatible oxygen ions sublattices. Electron mobilities more than one order of magnitude higher than those of hitherto-investigated perovskite-type interfaces are obtained. The spinel/perovskite twodimensional electron gas, where the two-dimensional conduction character is revealed by quantum magnetoresistance oscillations, is found to result from interface-stabilized oxygen vacancies confined within a layer of $0.9 \mathrm{~nm}$ in proximity to the interface. Our findings pave the way for studies of mesoscopic physics with complex oxides and design of high-mobility all-oxide electronic devices.

\footnotetext{
${ }^{1}$ Department of Energy Conversion and Storage, Technical University of Denmark, Risø Campus, 4000 Roskilde, Denmark. ${ }^{2}$ Nano-Science Center, Department of Chemistry, University of Copenhagen, 2100 Copenhagen, Denmark. ${ }^{3}$ Institute of Physics, Chinese Academy of Sciences, Beijing 100190 , China. ${ }^{4}$ Department of Physics, Technical University of Denmark, 2800 Lyngby, Denmark. ${ }^{5}$ Center for Electron Nanoscopy, Technical University of Denmark, 2800 Lyngby, Denmark. ${ }^{6}$ Leibniz Institute for Solid State and Materials Research, IFW Dresden, D-01171 Dresden, Germany. ${ }^{7}$ Laboratoire de Photonique et de Nanostructures-CNRS, Route de Nozay, 91460 Marcoussis, France. ${ }^{8}$ Center for Quantum Devices and Nano-Science Center, Niels Bohr Institute, University of Copenhagen, 2100 Copenhagen, Denmark. Correspondence and requests for materials should be addressed to Y.Z.C (email: yunc@dtu.dk).
} 
$\mathrm{H}$ igh-mobility two-dimensional electron gases (2DEGs) confined in epitaxially grown semiconductor heterostructures form the basis of modern electronic and photonic devices, and have constituted the material basis for the development of quantum transport and mesoscopic physics, for example, the resultant discoveries of the integer and fractional quantum Hall effects ${ }^{1,2}$. Different from those in semiconductors, strongly correlated electrons in complex oxides with partially occupied $d$-orbitals give rise to a variety of extraordinary electronic properties, such as high-temperature superconductivity, colossal magnetoresistance, ferromagnetism, ferroelectricity and multiferroicity. Therefore, the high-mobility 2DEGs at atomically engineered complex oxide interfaces not only show promise for multifunctional all-oxide devices with probably even richer behaviour than that in bulk ${ }^{3-10}$, but would also provide a wealth of opportunities to study mesoscopic physics with strongly correlated electrons confined in nanostructures. Nevertheless, this requires a large-enough electron mobility, so that the characteristic lengths of the system, such as the mean free path or the phase coherence length, become sizeable with respect to the typical dimension of quantum devices.

The enhancement of electron mobilities for complex oxide 2DEGs, however, meets formidable challenges. To date, these 2DEGs have been fabricated exclusively at oxide interfaces between perovskite bilayers ${ }^{4}$, such as the (001)-oriented polar $\mathrm{LaAlO}_{3}$ (LAO) films grown epitaxially on (001)-oriented nonpolar $\mathrm{SrTiO}_{3}$ (STO) single crystals with a $\mathrm{TiO}_{2}$ termination ${ }^{3}$. The two-dimensional (2D) electron mobility in these perovskite-type oxide interfaces is typically $\sim 1,000 \mathrm{~cm}^{2} \mathrm{~V}^{-1} \mathrm{~s}^{-1}$ at $2 \mathrm{~K}$ (refs 4,10 ), with a sheet carrier density, $n_{\mathrm{s}}$, being $10^{13}-10^{14} \mathrm{~cm}^{-2}$. This Hall mobility is still much lower than those for threedimensional oxygen-deficient STO single crystals ${ }^{11}$ and La-doped STO epitaxial films ${ }^{12}$, amounting to $1.3 \times 10^{4}$ and $3.2 \times$ $10^{4} \mathrm{~cm}^{2} \mathrm{~V}^{-1} \mathrm{~s}^{-1}$, respectively. The 2DEGs at these perovskitetype oxide interfaces are suggested to result from electronic reconstructions due to a polar discontinuity at the interface ${ }^{3}$; however, mechanisms such as ion transfer across the interface and formation of defects have also been identified to have important roles on the transport properties ${ }^{13,14}$. Harnessing the impurities and defects at these polar complex oxide interfaces remains elusive ${ }^{15}$. Despite deliberate efforts, the highest electron Hall mobility observed in the LAO/STO-based oxide interfaces is limited to the order of $5,000 \mathrm{~cm}^{2} \mathrm{~V}^{-1} \mathrm{~s}^{-1}$ at $2 \mathrm{~K}$ (refs 16,17 ). Besides interface polarity, we have recently found that chemical redox reactions at the oxide interface between STO single crystals and other complex oxides containing $\mathrm{Al}, \mathrm{Ti}, \mathrm{Zr}$ and $\mathrm{Hf}$ elements can provide an alternative approach to creating 2DEGs in complex oxide heterostructures ${ }^{18}$. Nevertheless, establishing electron confinement with increased carrier mobilities in STO-based heterointerfaces remains a challenge ${ }^{18}$.

Here we present a novel 2DEG with electron Hall mobilities as large as $1.4 \times 10^{5} \mathrm{~cm}^{2} \mathrm{~V}^{-1} \mathrm{~s}^{-1}$ and $n_{\mathrm{s}}$ as high as $3.7 \times$ $10^{14} \mathrm{~cm}^{-2}$ at $2 \mathrm{~K}$ by creating a spinel/perovskite complex oxide interface between epitaxial alumina $\left(\mathrm{Al}_{2} \mathrm{O}_{3}\right)$ films and STO single crystals (Fig. 1a). To our knowledge, it is the first time that complex oxide interfaces based on STO are found to exhibit carrier mobilities larger than any yet reported for either electrondoped STO single crystals ${ }^{11}$ or optimized epitaxial doped STO films ${ }^{12}$. Moreover, such a high mobility opens the door to the design of mesoscopic quantum devices based on complex oxides.

\section{Results}

Subunit cell layer-by-layer growth of $\boldsymbol{\gamma}-\mathrm{Al}_{\mathbf{2}} \mathrm{O}_{3}$ films. $\mathrm{Al}_{2} \mathrm{O}_{3}$ is a widely used oxide and is also one of the best insulating materials in nature, with a band gap normally above $8.0 \mathrm{eV}$. The synthesis of nanoscale $\mathrm{Al}_{2} \mathrm{O}_{3}$ usually results in $\gamma-\mathrm{Al}_{2} \mathrm{O}_{3}$ with a spinel-type structure, rather than the common $\alpha-\mathrm{Al}_{2} \mathrm{O}_{3}$ with a corundum structure, because the $\gamma-\mathrm{Al}_{2} \mathrm{O}_{3}$ has a lower surface energy than $\alpha-\mathrm{Al}_{2} \mathrm{O}_{3}$ (ref. 19). Remarkably, as illustrated in Fig. $1 \mathrm{~b}-\mathrm{d}$, despite differences in cation sublattices, the oxygen sublattice of the spinel $\gamma-\mathrm{Al}_{2} \mathrm{O}_{3}$ matches closely with that of the perovskite STO, as the lattice parameter of $\gamma-\mathrm{Al}_{2} \mathrm{O}_{3}$ is twice that of STO $\left(a_{\mathrm{STO}}=3.905 \AA, a_{\gamma-\mathrm{Al} 2 \mathrm{O} 3}=7.911 \AA\right.$ (ref. 20$)$, lattice mismatch of $1.2 \%)$. Such an excellent lattice match between oxygen sublattices, together with the low surface energy of $\gamma-\mathrm{Al}_{2} \mathrm{O}_{3}$, makes it compatible to grow epitaxially $\gamma-\mathrm{Al}_{2} \mathrm{O}_{3} / \mathrm{STO}$ spinel/perovskite heterostructures in a persistent $2 \mathrm{D}$ layer-by-layer growth mode (see Supplementary Fig. S1). Figure 1e shows typical intensity oscillations of the reflection high-energy electron diffraction (RHEED) pattern during the growth of a 3-unit cell (uc) $\gamma-\mathrm{Al}_{2} \mathrm{O}_{3}$ film at a growth temperature of $600^{\circ} \mathrm{C}$. For the epitaxial growth of ionic oxides, when all film components are supplied simultaneously, the oscillation period corresponds to the minimum unit of the chemical composition needed to ensure charge neutrality ${ }^{21-23}$. For $\gamma-\mathrm{Al}_{2} \mathrm{O}_{3}$ grown along the (001) direction, one intensity oscillation corresponds to the growth of one quarter unit cell film (Fig. 1e), as the $\gamma-\mathrm{Al}_{2} \mathrm{O}_{3}$ unit cell consists of four neutral ' $\mathrm{AlO}_{\mathrm{x}}$ ' subunit cells with an interlayer distance of about $0.2 \mathrm{~nm}$. Similar subunit cell layer-by-layer film growth has been observed in the epitaxial growth of spinel magnetite $\left(\mathrm{Fe}_{3} \mathrm{O}_{4}\right)^{24}$. The persistent layer-by-layer, $2 \mathrm{D}$ film growth results in a high-quality cubic-on-cubic $\gamma-\mathrm{Al}_{2} \mathrm{O}_{3} / \mathrm{STO}$ epitaxial heterointerface with no obvious dislocations as confirmed by scanning transmission electron microscopy (STEM) (Fig.1f,g).

Electrical transport properties of $\gamma-\mathrm{Al}_{2} \mathrm{O}_{3} / \mathrm{STO}$ interfaces. The investigation of conductivity in our $\gamma-\mathrm{Al}_{2} \mathrm{O}_{3} / \mathrm{STO}$ heterostructures shows that the interface between the two insulators can become metallic with electrons as the dominant charge carriers (see Supplementary Fig. S2). Of note, under the condition of our film growth, the bare STO substrate remains highly insulating without film deposition. More strikingly, 2DEGs with extremely high Hall electron mobilities are obtained when the $\gamma-\mathrm{Al}_{2} \mathrm{O}_{3}$ film is grown at an oxygen background pressure of $10^{-4}$ mbar and a growth temperature of $600^{\circ} \mathrm{C}$ (Fig. $2 \mathrm{a}-\mathrm{c}$ ). As shown in Fig. 2, the interfacial conduction depends critically on the thickness, $d$, of the $\gamma-\mathrm{Al}_{2} \mathrm{O}_{3}$ film. The heterointerface changes from highly insulating to metallic when $d$ is above a threshold thickness of approximately $2 \mathrm{uc}$ (Fig. 2d,e). At $d=2 \mathrm{uc}$, the interface shows a sheet resistance, $R_{\mathrm{s}}$, and a carrier density, $n_{\mathrm{s}}$, in the order of $10 \mathrm{k} \Omega / \square$ and $2.3 \times 10^{13} \mathrm{~cm}^{-2}$ at $T=300 \mathrm{~K}$, respectively, similar to the perovskite-type LAO/STO interface ${ }^{4,6-9,14,17}$. Remarkably, we find a striking $R_{\mathrm{s}}$ decrease of about three orders in magnitude and a Hall mobility as high as $\mu_{\mathrm{Hall}} \sim 1.1 \times 10^{4} \mathrm{~cm}^{2} \mathrm{~V}^{-1} \mathrm{~s}-1$ at $T=2 \mathrm{~K}$ in the spinel/perovskite $\gamma-\mathrm{Al}_{2} \mathrm{O}_{3} / \mathrm{STO}$ interface. By carefully controlling the film growth down to a subunit cell level, a great $R_{\mathrm{s}}$ decrease of approximately four orders in magnitude is observed at $d=2.5 \mathrm{uc}$, which is accompanied by the presence of non-linear Hall resistance with respect to magnetic fields at temperatures below $100 \mathrm{~K}$ (see Supplementary Fig. S3). A linear fitting to the low-field Hall resistance gives rise to an impressive $\mu_{\text {Hall }}$ of approximately $1.4 \times 10^{5} \mathrm{~cm}^{2} \mathrm{~V}^{-1} \mathrm{~s}-1$, with an $n_{\mathrm{s}}$ of $3.7 \times 10^{14} \mathrm{~cm}^{-2}$ at $2 \mathrm{~K}$, which is consistent with those obtained by fitting the entire non-linear Hall effect within a twoband model (see Supplementary Fig. S3). Note that the highmobility 2DEGs with $\mu_{\text {Hall }} \geq 10^{4} \mathrm{~cm}^{2} \mathrm{~V}^{-1} \mathrm{~s}^{-1}$ at $T=2 \mathrm{~K}$ are only detected in the thickness range of 2 uc $\leq d<3$ uc. Further increasing $d$ deteriorates the electron mobility to less than $1,000 \mathrm{~cm}^{2} \mathrm{~V}^{-1} \mathrm{~s}^{-1}$, probably due to the significant outward 
a

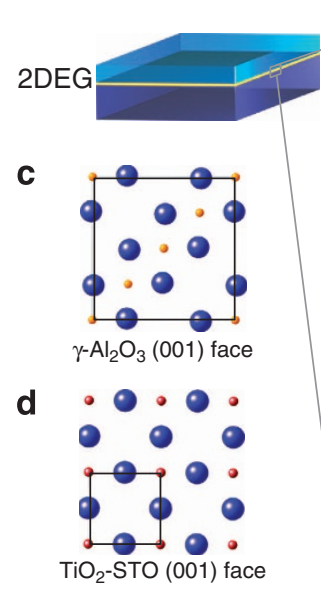

b

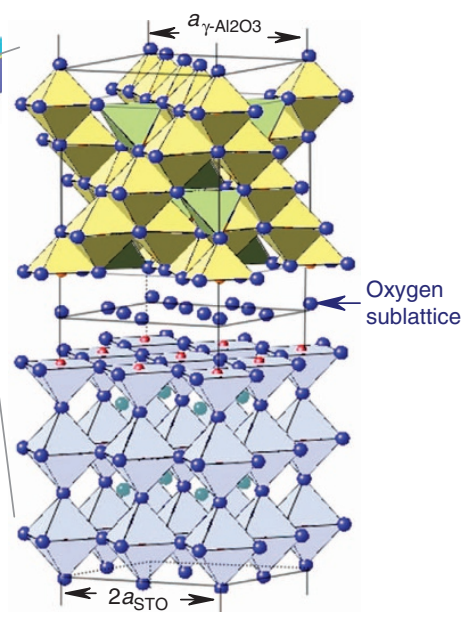

e

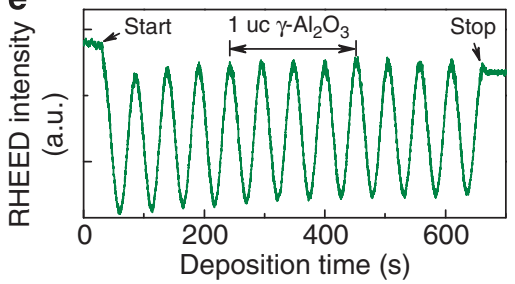

f
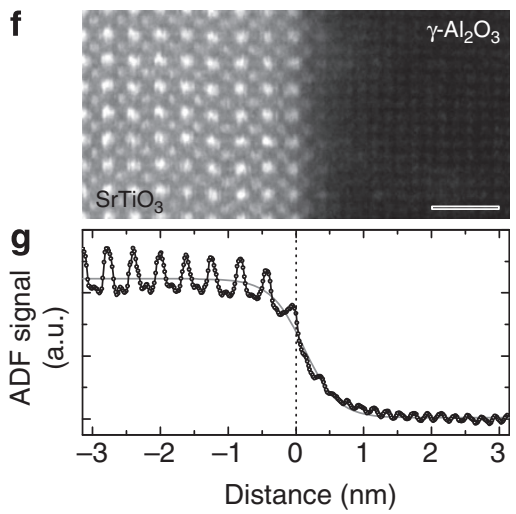

Figure 1 | High-mobility 2DEGs at epitaxial spinel/perovskite $\gamma-\mathbf{A l}_{\mathbf{2}} \mathbf{O}_{\mathbf{3}} / \mathbf{S T O}$ interfaces. (a) A sketch of the heterostructure. (b) Oxygen sublattices as the backbone to build the spinel/perovskite heterostructure. The compatibility in oxygen sublattices of a $\gamma-\mathrm{Al}_{2} \mathrm{O}_{3}$ surface and the TiO $\mathrm{O}_{2}$-terminated STO surface is shown in $\mathbf{c}$ and $\mathbf{d}$, respectively. Of note, the tetrahedral cation sites in $\gamma-\mathrm{Al}_{2} \mathrm{O}_{3}$ are not shown. (e) Typical RHEED intensity oscillations for the growth of a 3-uc $\gamma-\mathrm{Al}_{2} \mathrm{O}_{3}$ on STO in a subunit cell layer-by-layer mode. (f) HAADF STEM image of the epitaxial $\gamma-\mathrm{Al}_{2} \mathrm{O}_{3} / \mathrm{STO}$ interface. Scale bar, $1 \mathrm{~nm}$. Sr ions are brightest, followed by Ti. The faintly visible Al elements can be determined by the averaged line profiles across the interface shown in $\mathbf{g}$. A well-developed $\mathrm{TiO}_{2}-\mathrm{AlO}_{\mathbf{x}}$ heterointerface is defined.
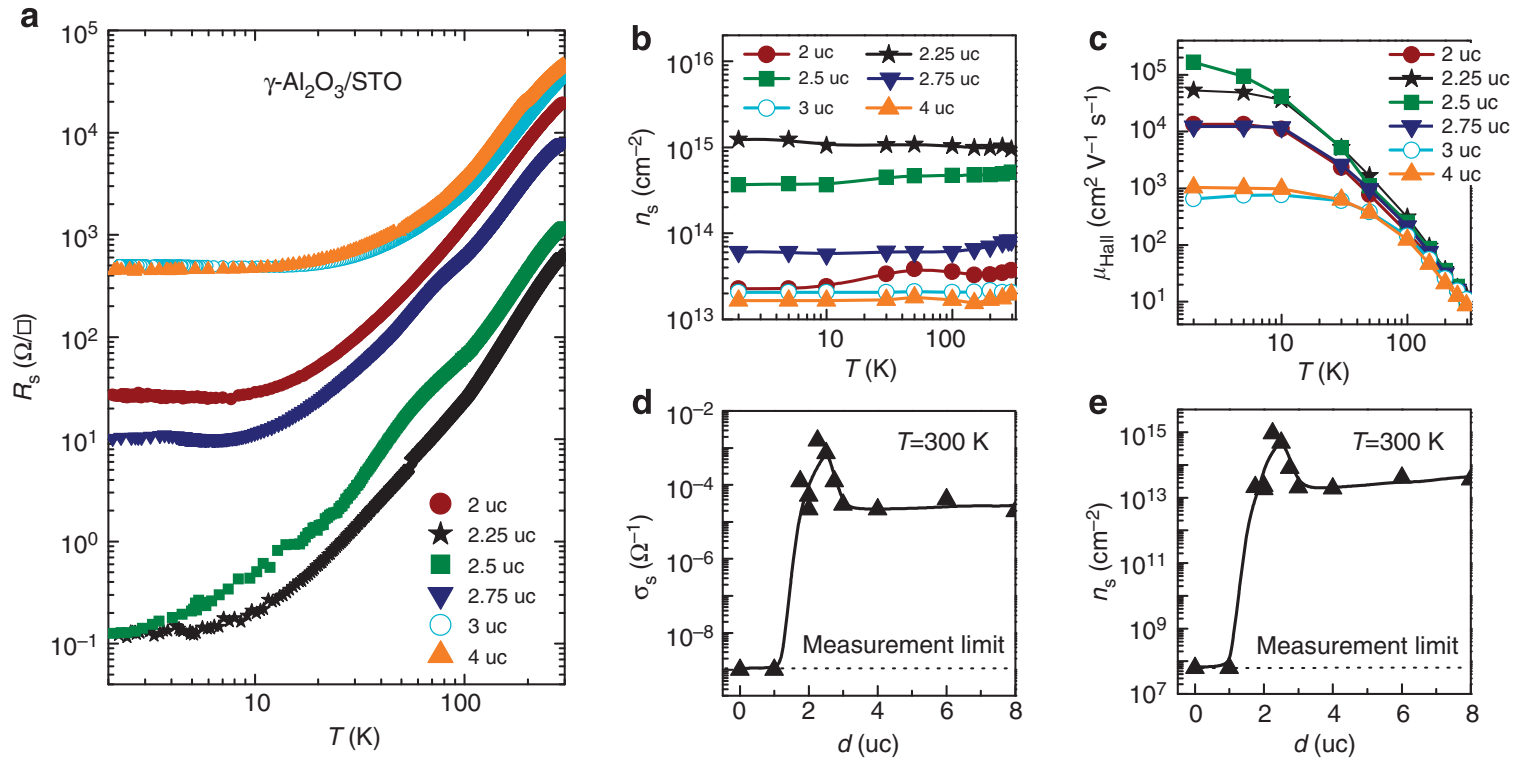

Figure 2 | Thickness-dependent electronic properties of the $\gamma-\mathbf{A l}_{\mathbf{2}} \mathbf{O}_{\mathbf{3}} / \mathbf{S T O}$ interface. (a-c) Temperature dependence of sheet resistance, $R_{\mathrm{s}}$, carrier density, $n_{\mathrm{s}}$ and low-field electron Hall mobility, $\mu_{\text {Hall }}$, for the interface conduction at different film thicknesses. (d,e) Thickness dependence of the sheet conductance, $\sigma_{\mathrm{s}}$ and $n_{\mathrm{s}}$ measured at $300 \mathrm{~K}$. High-mobility $2 \mathrm{DEG}$ are obtained at a thickness range of 2 uc $\leq d<3$ uc. The lines are guides to the eye.

diffusion of the Ti-cations across the interface as observed by electron energy-loss spectroscopy (EELS; see Supplementary Fig. S4).

2D quantum oscillations of the conduction in $\gamma-\mathrm{Al}_{2} \mathrm{O}_{3} / \mathrm{STO}$. The 2D nature of the conduction in our spinel/perovskite heterostructures is indicated by angle-dependent Shubnikov-de Haas
( $\mathrm{SdH})$ quantum oscillations, which are superimposed on a huge background of positive magnetoresistance (Fig. 3a). After subtracting the magnetoresistance background, the $\mathrm{SdH}$ oscillations become apparent (Fig. 3b) and the extrema positions show a cosine dependence with the angle $\theta$ between the magnetic field and the surface normal (Fig. 3c). This reveals the 2D nature of the electron gas formed at our $\gamma-\mathrm{Al}_{2} \mathrm{O}_{3} / \mathrm{STO}$ interfaces. Besides, the 
a

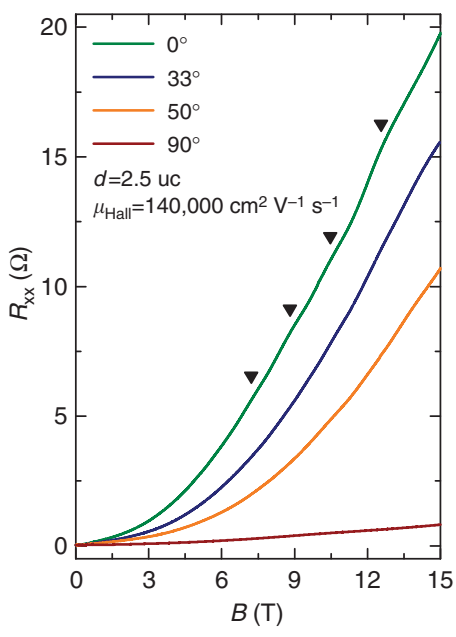

b

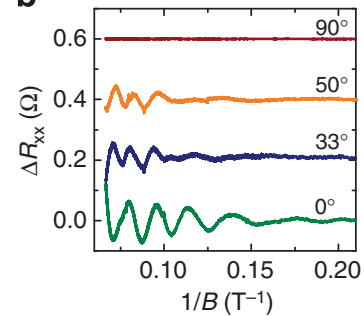

C

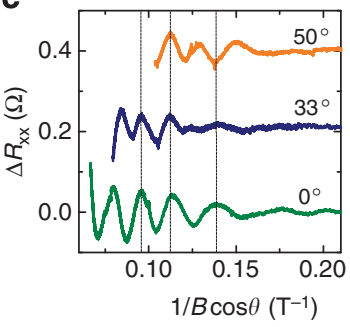

Figure 3 | 2D quantum oscillations of the conduction at $\gamma-\mathrm{Al}_{2} \mathrm{O}_{3} / \mathrm{STO}$ interfaces. (a) Longitudinal resistance, $R_{\mathrm{x} x}$ as a function of magnetic field with visible $\mathrm{SdH}$ oscillations (arrowheads) under different tilt angle, $\theta$, at $0.3 \mathrm{~K}$ for the $d=2.5$ uc sample. (b,c) Amplitude of the $\mathrm{SdH}$ oscillations, $\Delta R_{\mathrm{xx}}$, under different $\theta$ versus the reciprocal total magnetic field and the reciprocal perpendicular magnetic field component, respectively. The $\mathrm{SdH}$ oscillations depend mainly on the reciprocal perpendicular magnetic field component, particularly in the $\theta$ angle of $0^{\circ}-33^{\circ}$, which suggests a $2 \mathrm{D}$ conduction nature of the $\gamma-\mathrm{Al}_{2} \mathrm{O}_{3} /$ STO interface.

absence of oscillations at $\theta=90^{\circ}$ further confirms that the spatial width of the $2 \mathrm{DEG}$ is smaller than at least the cyclotron radius at $15 \mathrm{~T}$, the typical value of which is below $10 \mathrm{~nm}$ for our heterostructures. Moreover, the angular dependence of the SdH oscillations measured at high magnetic fields suggests a multiplesubband contribution to charge transport. For instance, an extra feature is observed at $\theta=50^{\circ}$ with $B \cos \theta=7.2 \mathrm{~T}$, which may result from a $\pi$ shift of the oscillations due to a spin-split band. Such a phase shift has been observed in the high-mobility 2DEG of GaN/AlGaN interfaces when the Zeeman energy (depending on the total $B$ ) and the cyclotron energy (depending on the perpendicular component of $B$ ) are equal ${ }^{25}$.

To confirm the high mobility achieved in our $\gamma-\mathrm{Al}_{2} \mathrm{O}_{3} / \mathrm{STO}$ 2DEGs, we increased the visibility of the SdH oscillations by cooling one sample $(d=2.25 \mathrm{uc})$ down to $22 \mathrm{mK}$ in a dilution refrigerator. Ultra-low noise measurements allow us to evidence the oscillations down to about $1 \mathrm{~T}$ (Fig. 4a), which directly shows that the quantum mobility extracted from the $\mathrm{SdH}$ oscillations, $\mu_{\mathrm{SdH}}$, is in the range of $10^{4} \mathrm{~cm}^{2} \mathrm{~V}^{-1} \mathrm{~s}^{-1}$, as inferred from the onset of oscillations. Importantly, the low-field dependence of the $\mathrm{SdH}$ oscillations reveals the typical behaviour due to a single band. According to theory ${ }^{26}$, the oscillations amplitude $\Delta R_{\mathrm{xx}}$ can be described as:

$$
\Delta R_{x x}=4 R_{0} e^{-\alpha T_{D}} \alpha T / \sinh (\alpha T)
$$

where, $\alpha=2 \pi^{2} k_{\mathrm{B}} / \hbar \omega_{c}, \omega_{c}=e B / m^{*}$ is the cyclotron frequency, $m^{*}$ is the carrier effective mass, $k_{\mathrm{B}}$ is Boltzmann's constant and $\hbar$ is Planck's constant divided by $2 \pi$. $R_{0}$ is the classical resistance in zero field. $T_{D}=\hbar / 2 \pi k_{\mathrm{B}} \tau$ is the Dingle temperature, $\tau$ is the total scattering time. At a fixed magnetic field, $m^{*}$ can be deduced by fitting the temperature-dependent oscillation amplitude with $\Delta R_{x x}(T) / \Delta R_{x x}\left(T_{0}\right)=T \sinh \left(\alpha T_{0}\right) / T_{0} \sinh (\alpha T)\left(T_{0}=22 \mathrm{mK}\right) . \quad$ As shown in Fig. $4 \mathrm{~b}$, for $B=2.04 \mathrm{~T}$ the fit leads to an effective mass of $m^{*}=(1.22 \pm 0.03) m_{\mathrm{e}}\left(m_{\mathrm{e}}\right.$ is the bare electron mass),

a
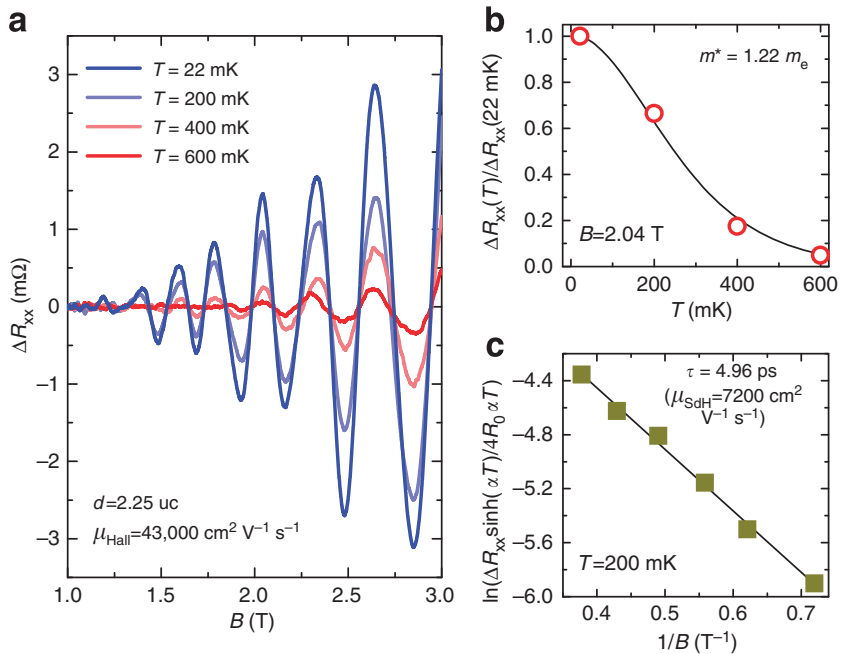

Figure 4 | Small-field and low-temperature behaviour of the SdH oscillations. (a) Temperature dependence of the SdH oscillations at $\theta=0$ for the $d=2.25$ uc sample. (b) Temperature dependence of the scaled oscillation amplitude at $B=2.04 \mathrm{~T}$, giving a carrier effective mass of $1.22 \mathrm{me}_{\mathrm{e}}$ (c) Dingle plot of the $\mathrm{SdH}$ oscillations at $200 \mathrm{mK}$, giving a total scattering time $\tau=4.96 \times 10^{-12} \mathrm{~s}$, a related Dingle temperature $T_{\mathrm{D}}=0.24 \mathrm{~K}$ and a consequent quantum mobility $\mu_{\mathrm{SdH}}=7.2 \times 10^{3} \mathrm{~cm}^{2} \mathrm{~V}^{-1} \mathrm{~s}^{-1}$.

consistent with those reported for other STO-based heterostructures $^{16,27-30}$. At a fixed temperature, $T_{\mathrm{D}}$ or $\tau$ can be deduced from the slope of the Dingle plot, that is, $\ln \left[\Delta R_{x x} \sinh (\alpha T) / 4 R_{0} \alpha T\right]$ versus $1 / B$ (Fig. $4 c$ for $T=200 \mathrm{mK}$ ), which gives a $\tau=4.96 \times 10^{-12} \mathrm{~s}$ or $T_{\mathrm{D}}=0.24 \mathrm{~K}$, corresponding to a quantum mobility $\mu_{\mathrm{SdH}}=e \tau / \mathrm{m}^{*}$ of $7.2 \times 10^{3} \mathrm{~cm}^{2} \mathrm{~V}^{-1} \mathrm{~s}^{-1}$. Such an unprecedented high $\mu_{\mathrm{SdH}}$ in our $\gamma-\mathrm{Al}_{2} \mathrm{O}_{3} / \mathrm{STO}$ 2DEGs is more than one order of magnitude higher than those observed in the perovskite/ perovskite $\mathrm{LAO} / \mathrm{STO}^{16,17,27}$ and $\mathrm{GaTiO}_{3} / \mathrm{STO}^{30}$ heterostructures, which are typically below $300 \mathrm{~cm}^{2} \mathrm{~V}^{-1} \mathrm{~s}^{-1}$. Note that the difference between $\mu_{\text {Hall }}$ and $\mu_{\text {SdH }}$ in our $\gamma-\mathrm{Al}_{2} \mathrm{O}_{3} / \mathrm{STO}$ heterostructures could come from a different scattering time (that is, the transport scattering time and the total scattering time, respectively), which has also been reported in the $\mathrm{LAO} / \mathrm{STO}^{16,17,27}$ and $\delta$-doped STO heterostructures $^{28,29}$, as well as the $\mathrm{GaAs} / \mathrm{AlGaAs}$ heterostructures ${ }^{31}$. In short, the SdH measurements support the formation of highmobility 2DEGs at our spinel/perovskite heterointerfaces (see also Supplementary Fig. S5).

Spatial confinement of the $\gamma-\mathrm{Al}_{2} \mathrm{O}_{3} / \mathrm{STO}$ interface 2DEG. To determine the origin and depth-profile for the conduction in the $\gamma-\mathrm{Al}_{2} \mathrm{O}_{3} / \mathrm{STO}$ heterostructures, angle-resolved X-ray photoelectron spectroscopy (XPS) measurements are performed. We find that the electrons are exclusively accumulated on the otherwise empty $3 d$ shell of $\mathrm{Ti}^{4+}$ on the STO side. The most remarkable XPS result is that the $\mathrm{Ti}^{3+}$ signal in $\gamma-\mathrm{Al}_{2} \mathrm{O}_{3} / \mathrm{STO}$ heterointerfaces shows strong dependence on the photoelectrons detection angle, $\varphi$, with respect to the surface normal. An increase of the $\mathrm{Ti}^{3+}$ signal with increasing $\varphi$, as shown in Fig. $5 \mathrm{a}$, is clearly detected for $d=2.5 \mathrm{uc}$ with the highest Hall mobility. This further confirms that the conduction in our $\gamma-\mathrm{Al}_{2} \mathrm{O}_{3} / \mathrm{STO}$ heterointerface is highly confined at the interface region. To make more quantitative analyses, we assume a simple case that the 2DEG extends from the interface to a depth, $t$, into the STO substrate ${ }^{32}$. The interface region is further assumed to be stoichiometric, sharp and characterized by a constant fraction, $p$, of $\mathrm{Ti}^{3+}$ per STO unit cell. Taking into account the attenuation length of photoelectrons, 


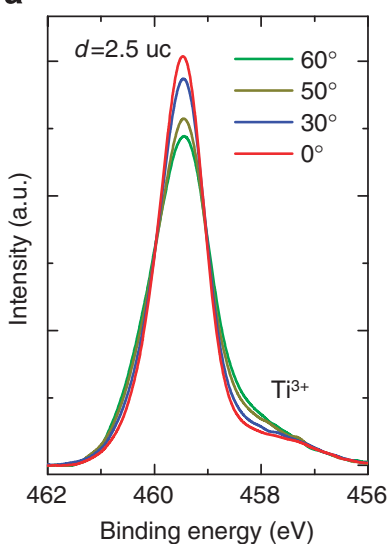

b

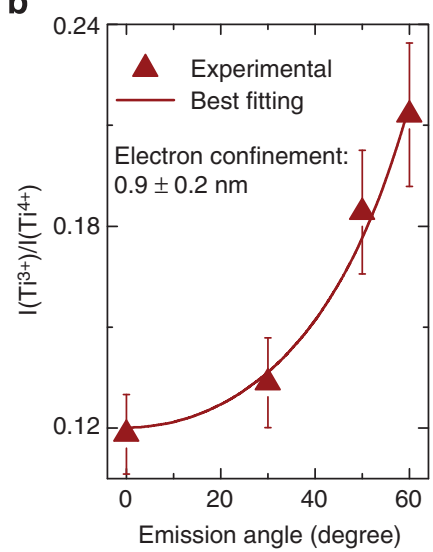

Figure 5 | Spatial confinement of the 2DEG at the $\gamma-\mathrm{Al}_{2} \mathrm{O}_{3} / \mathrm{STO}$ heterointerface determined by angle-resolved XPS. (a) The Ti $2 p_{3 / 2}$ XPS spectra at various emission angles $\varphi$ for the $d=2.5$ uc sample. (b) The angle dependence of the ratio of $\mathrm{Ti}^{3+}$ to $\mathrm{Ti}^{4+}$ signal, $I\left(\mathrm{Ti}^{3+}\right) / I\left(\mathrm{Ti}^{4+}\right)$, indicates a strong confinement of the conduction layer within $0.9 \mathrm{~nm}$. Error bars indicate deviations, $\pm 10 \%$, of experimental values.

the ratio of $\mathrm{Ti}^{3+}$ to $\mathrm{Ti}^{4+}$ signal, $I\left(\mathrm{Ti}^{3+}\right) / I\left(\mathrm{Ti}^{4+}\right)$, as a function of $\varphi$ can be calculated as ${ }^{32}$ :

$$
\frac{I\left(T i^{3+}\right)}{I\left(T i^{4+}\right)}=\frac{p[1-\exp (-t / \lambda \cos \varphi)]}{1-p[1-\exp (-t / \lambda \cos \varphi)]}
$$

where, $\lambda$ is the electron escape depth in STO. According to the NIST database (NIST Standard Reference Database 71, version $1.2), \lambda$ is approximately $2.2 \mathrm{~nm}$ for our setup. As shown in Fig. $5 \mathrm{~b}$, the best fitting of the experimental $I\left(\mathrm{Ti}^{3+}\right) / I\left(\mathrm{Ti}^{4+}\right)$ ratios gives a $p \sim 0.31$, which equals to an $n_{\mathrm{s}} \sim 2.1 \times 10^{14} \mathrm{~cm}^{-2}$ and a $t$ of $0.9 \mathrm{~nm}$. Therefore, the electrons at our $\gamma-\mathrm{Al}_{2} \mathrm{O}_{3} / \mathrm{STO}$ heterointerface are strongly confined within approximately the first 2 uc of STO surface in proximity to the interface. Note that the $n_{\mathrm{s}}$ deduced here is slightly lower than that obtained from Hall data (Fig. 2c). This could be due to the presence of outward diffusion of the Ti-cations into alumina films, where $\mathrm{Ti}^{4}+$ is the dominant component (see Supplementary Figure S4). Such concern is also consistent with the fact that the out-diffused $\mathrm{Ti}$ is found to have a negligible contribution to the measured interface conduction. For example, the interface conduction remains unaffected when the capping alumina film is etched away by a $4-\mathrm{M}$ aqueous $\mathrm{NaOH}$ solution. This strongly suggests that the effective charge carriers are mainly located on the STO side.

\section{Discussion}

As each layer of the (001)-oriented $\gamma-\mathrm{Al}_{2} \mathrm{O}_{3} / \mathrm{STO}$ heterointerface is nominally charge neutral, the polar discontinuity-induced electronic reconstruction as expected in the LAO/STO interface ${ }^{3}$ may not contribute here. The presence of $\mathrm{Ti}^{3+}$ is probably a signature of the formation of oxygen vacancies on the STO side. This scenario is consistent with the fact that the interfacial conductivity can be completely removed when the $\mathrm{Ti}^{3+}$ content is significantly suppressed by suitable annealing in 1 bar pure $\mathrm{O}_{2}$ at a temperature higher than $200^{\circ} \mathrm{C}$ (see Supplementary Fig. S6). Such an oxygen-vacancy-dominated 2DEG is expected to be formed as a consequence of chemical redox reactions occurring on the STO surface during the film growth of $\gamma-\mathrm{Al}_{2} \mathrm{O}_{3}$, analogous to what has been observed in metallic amorphous STO-based heterostructures grown at room temperature ${ }^{18}$. Note that the

2DEG at the crystalline $\gamma-\mathrm{Al}_{2} \mathrm{O}_{3} / \mathrm{STO}$ heterointerface is formed at a high temperature of $600^{\circ} \mathrm{C}$, where the oxygen ions in STO are already highly mobile. This is normally expected to level out any difference in the depth-profile of oxygen distribution in $\mathrm{STO}^{18,33}$. However, this is not the case in the crystalline $\gamma-\mathrm{Al}_{2} \mathrm{O}_{3} / \mathrm{STO}$ heterostructures as inferred from both Figs 3 and 5. Moreover, the conduction at the interface of thick films, for example, at $d=8 \mathrm{uc}$, can survive the annealing at $300^{\circ} \mathrm{C}$ for $24 \mathrm{~h}$ in $1 \mathrm{bar}$ pure $\mathrm{O}_{2}$ with only negligible changes in the conductivity (see Supplementary Fig. S6). These features strongly suggest that the oxygen vacancies and the 2DEGs are stabilized by an interface effect, such as by the formation of a space charge region near the heterointerface. It is worth noting that an inherent oxygen ion deficiency has been observed at the grain boundary of STO bicrystals $^{34}$, where a considerable electron accumulation has also been predicted if the barrier height of the grain boundary is deliberately controlled ${ }^{35}$. The high electron mobility of STObased oxide materials at low temperatures is generally related to the polarization shielding of the ionized defect scattering centres driven by the large dielectric constant of $\mathrm{STO}^{36}$. The higher mobility of our spinel/perovskite oxide interface compared with the perovskite-type oxide heterointerface may be due to the better lattice match and, thereby, a more perfect structure and welldefined interface. Though further investigations are needed to reveal how the interface properties increase the mobility and the associated strong suppression of the defect and impurity scattering, our results strongly suggest that defect engineering of oxygen vacancies is crucial for the high mobility of 2DEGs confined at the interface between complex oxides.

In conclusion, we have demonstrated that high-mobility 2DEGs with clear quantum magnetoresistance oscillations and strong spatial confinement can be created at well-defined spinel/ perovskite $\gamma-\mathrm{Al}_{2} \mathrm{O}_{3} / \mathrm{STO}$ oxide interfaces. The strongly spatial confinement of charge carriers achieved directly in the asdeposited spinel/perovskite oxide heterostructures without any post annealing provides the possibility to fabricate multilayers of complex oxides with several 2DEGs. Furthermore, by combining two of the largest groups of oxides, plenty of new physical properties, for instance, interfacial magnetism ${ }^{6}$ and superconductivity $^{7}$ as observed in the perovskite-type LAO/STO interface, may be found at the $\gamma-\mathrm{Al}_{2} \mathrm{O}_{3} / \mathrm{STO}$ heterointerface. Finally, with a large enhancement of the electron mobility, the $\gamma-\mathrm{Al}_{2} \mathrm{O}_{3} / \mathrm{STO}$ heterointerface probably enables the design of mesoscopic quantum devices based on complex oxide 2DEGs and opens new avenues for oxide nanoelectronics and mesoscopic physics.

\section{Methods}

Sample growth. The $\gamma-\mathrm{Al}_{2} \mathrm{O}_{3}$ thin films were grown by pulsed laser deposition ${ }^{37}$ using a $\mathrm{KrF}$ laser $(\lambda=248 \mathrm{~nm})$ with a repetition rate of $1 \mathrm{~Hz}$ and laser fluence of $1.5 \mathrm{~J} \mathrm{~cm}^{-2}$. The target-substrate distance was fixed at $5.6 \mathrm{~cm}$. Commercial $\alpha-\mathrm{Al}_{2} \mathrm{O}_{3}$ single crystals were used as targets. Singly $\mathrm{TiO}_{2}$-terminated (001) STO crystals with a size of $5 \times 5 \times 0.5 \mathrm{~mm}^{3}$ were used as substrates. Note that the $\mathrm{TiO}_{2}$ termination of our substrates is obtained by chemical etching using $\mathrm{HCL}-\mathrm{HNO}_{3}$ as acidic solution $^{37}$, which is found to produce less defects on the STO surface compared with the conventional buffered hydrofluoric acid etch method ${ }^{38,39}$. The film growth process was monitored by in-situ high pressure RHEED. During deposition, the oxygen pressure was fixed at $10^{-4}$ mbar with the deposition temperature changing from room temperature $\left(20^{\circ} \mathrm{C}\right)$ to $700{ }^{\circ} \mathrm{C}$. After film deposition, the samples were cooled down to room temperature at the deposition pressure. The film thickness was determined by both RHEED oscillations and X-ray reflectivity measurements.

Electrical transport measurement. The transport properties of the buried inter face were measured using a four-probe Van der Pauw method, with ultrasonically wire-bonded aluminium wires as electrodes, placed at the corners of the square sample. The temperature-dependent electrical transport and Hall-effect measurements were performed in a CRYOGENIC cryogen-free measurement system, with the temperature ranging from $300 \mathrm{~K}$ down to $2 \mathrm{~K}$ and magnetic fields up to $16 \mathrm{~T}$. To confirm the carrier density and mobility, some Hall-bar patterned samples were 
also measured, which were prepared directly through a mechanical mask ${ }^{18}$. Note that the use of a mechanical mask at deposition temperatures higher than $500{ }^{\circ} \mathrm{C}$ may have a deleterious effect on the carrier mobility, as the high oxygen ion diffusion can unintentionally disturb the oxygen equilibrium for realizing high mobility. The angle-dependent $\mathrm{SdH}$ measurements were performed in a sorptionpumped ${ }^{3} \mathrm{He}$ cryostat with standard lock-in technique at $0.3 \mathrm{~K}$, with magnetic fields up to $15 \mathrm{~T}$ by changing the angles manually. The temperature-dependent $\mathrm{SdH}$ measurements were performed in a dilution refrigerator with a base temperature of $22 \mathrm{mK}$ and an improved temperature stability, using ultra-low noise electronics. During all the transport measurements, the applied currents were within $1-10 \mu \mathrm{A}$ (for AC current, the frequency was $327 \mathrm{~Hz}$ ). Special care was taken to avoid heating effect.

XPS measurement. The XPS measurements were performed in a Kratos Axis Ultra ${ }^{\mathrm{DLD}}$ instrument, using a monochromatic $\mathrm{Al} \mathrm{K} \alpha \mathrm{X}$-ray source with photon energy of $1,486.6 \mathrm{eV}$. This leads to a kinetic energy of Ti $2 p$ electrons of roughly $1,025 \mathrm{eV}$. According to the NIST database(NIST Standard Reference Database 71, version 1.2), the electron escape depth is approximately $22 \AA$ in STO at this kinetic energy. The pass energy used for the high resolution scan was $20 \mathrm{eV}$. The detection angle of the electrons varied between $0^{\circ}$ and $60^{\circ}$ with respect to the sample normal. For analysing the Ti $2 p_{3 / 2}$ peaks $\left(\mathrm{Ti}^{4+}\right.$ is at a binding energy of $459.5 \mathrm{eV}$, whereas the $\mathrm{Ti}^{3+}$ is $1.6 \mathrm{eV} \pm 0.1 \mathrm{eV}$ lower), a Shirley background was subtracted and the spectra were normalized to the total area below the Ti peaks $\left([\mathrm{Ti}]=\left[\mathrm{Ti}^{4+}\right]+\left[\mathrm{Ti}^{3+}\right]=100 \%\right)$.

STEM and EELS measurements. Aberration-corrected STEM measurements were performed by an FEI Titan 80-300ST TEM equipped with a high brightness Shottky emitter (XFEG) and a Gatan Image Filter (Tridiem). High-angle annular dark field (HAADF) images were acquired at $300 \mathrm{kV}$, where the probe size, convergence angle and HAADF collection angle were $0.8-1 \AA$, $20 \mathrm{mrad}$ and $46-291 \mathrm{mrad}$, respectively. For EELS in the STEM, an accelerating voltage of $120 \mathrm{kV}$ (probe size of $1.5-2.0 \AA$ ) was used to reduce knock-on damage to the specimen. The energy resolution of EELS was $\sim 0.9 \mathrm{eV}$. Spectrum imaging was used to collect spectra across the interface. We typically recorded the spectrum images consisting of 40 ten-analysis point lines (that is, $10 \times 40$ pixel) parallel to the interface and acquired each line by an increment of $0.28 \mathrm{~nm}$. Each spectrum was obtained at a dispersion of $0.1 \mathrm{eV}$ for $0.2-0.4 \mathrm{~s}$. Then the spectra along the lines were summed after removing the spectra from beam-damaged regions according to the HAADF contrast to increase signal/background ratio.

\section{References}

1. Klitzing, K. v., Dorda, G. \& Pepper, M. New methods for high-accuracy determination of the fine-structure constant based on quantized Hall resistance. Phys. Rev. Lett. 45, 494-497 (1980).

2. Tsui, D. C., Stormer, H. L. \& Gossard, A. C. Two-dimensional magnetotransport in the extreme quantum limit. Phys. Rev. Lett. 48, 1559-1562 (1982).

3. Ohtomo, A. \& Hwang, H. Y. A high-mobility electron gas at the $\mathrm{LaAlO}_{3} /$ $\mathrm{SrTiO}_{3}$ heterointerface. Nature 427, 423-426 (2004).

4. Mannhart, J. \& Schlom, D. G. Oxide interfaces: an opportunity for electronics. Science 327, 1607-1611 (2010).

5. Irvin, P. et al. Rewritable nanoscale oxide photodetector. Nat. Photon. 4, 849-852 (2010).

6. Brinkman, A. et al. Magnetic effects at the interface between non-magnetic oxides. Nat. Mater. 6, 493-496 (2007).

7. Reyren, N. et al. Superconducting interfaces between insulating oxides. Science 317, 1196-1199 (2007).

8. The interface is still the device. Nat. Mater. 11, 91 (2012).

9. Chakhalian, J., Millis, A. J. \& Rondinelli, J. Whither the oxide interface. Nat. Mater. 11, 92-94 (2012).

10. Park, J. W. et al. Creation of a two-dimensional electron gas at an oxide interface on silicon. Nat. Commun. 1, 94 (2010).

11. Frederikse, H. P. R. \& Hosler, W. R. Hall mobility in $\mathrm{SrTiO}_{3}$. Phys. Rev. 161, 822-827 (1967)

12. Son, J. et al. Epitaxial $\mathrm{SrTiO}_{3}$ films with electron mobilities exceeding $30000 \mathrm{~cm}^{2} \mathrm{~V}^{-1} \mathrm{~s}^{-1}$. Nat. Mater. 9, 482-484 (2010).

13. Willmott, P. R. et al. Structural basis for the conducting interfaces between $\mathrm{LaAlO}_{3}$ and $\mathrm{SrTiO}_{3}$. Phys. Rev. Lett. 99, 155502 (2007).

14. Kalabukhov, A. et al. Effect of oxygen vacancies in the $\mathrm{SrTiO}_{3}$ substrate on the electrical properties of the $\mathrm{LaAlO}_{3} / \mathrm{SrTiO}_{3}$ interface. Phys. Rev. B 75, 121404 (2007).

15. Chambers, S. A. Understanding the mechanism of conductivity at the $\mathrm{LaAlO}_{3} /$ $\mathrm{SrTiO}_{3}$ (001) interface. Surface Sci. 605, 1133-1140 (2011).

16. Caviglia, A. D. et al. Two-dimensional quantum oscillations of the conductance at $\mathrm{LaAlO}_{3} / \mathrm{SrTiO}_{3}$ interfaces. Phys. Rev. Lett. 105, 236802 (2010).

17. Huijben, M. et al. High mobility interface electron gas by defect scavenging in a modulation doped oxide heterostructure. Preprint at http://arXiv:1008.1896v1 (2010).

18. Chen, Y. Z. et al. Metallic and insulating interfaces of amorphous $\mathrm{SrTiO}_{3}$-based oxide heterostructures. Nano Lett. 11, 3774-3778 (2011).
19. McHale, J. M., Auroux, A., Perrotta, A. J. \& Navrotsky, A. Surface energies and thermodynamic phase stability in nanocrystalline aluminas. Science 277, 788-791 (1997).

20. Zhou, R. S. \& Snyder, R. L. Structures and transformation mechanisms of the $\eta$, $\gamma$ and $\theta$ transition aluminas. Acta Cryst. B 47, 617-630 (1991).

21. Terashima, T. et al. Reflection high-energy electron diffraction oscillations during epitaxial growth of high-temperature superconducting oxides. Phys. Rev. Lett. 65, 2684-2687 (1990).

22. Rijnders, A. J. H. M. The initial growth of complex oxides: study and manipulation. PhD thesis. Univ. Twente (2001).

23. Barber, Z. H. The control of thin film deposition and recent developments in oxide film growth. J. Mater. Chem. 16, 334-344 (2006).

24. Reisinger, D. et al. Sub-unit cell layer-by-layer growth of $\mathrm{Fe}_{3} \mathrm{O}_{4}, \mathrm{MgO}$, and $\mathrm{Sr}_{2} \mathrm{RuO}_{4}$ thin films. Appl. Phys. A 77, 619-621 (2003).

25. Knap, W. et al. Spin and interaction effects in Shubnikov-de Haas oscillations and the quantum Hall effect in GaN/AlGaN heterostructures. J. Phys. Condens. Mat. 16, 3421-3432 (2004).

26. Shoenberg, D. Magnetic Oscillations in Metals (Cambridge Univ. Press, Cambridge, England, 1984).

27. Ben Shalom, M., Ron, A., Palevski, A. \& Dagan, Y. Shubnikov-de Haas oscillations in $\mathrm{SrTiO}_{3} / \mathrm{LaAlO}_{3}$ interface. Phys. Rev. Lett. 105, 206401 (2010).

28. Jalan, B., Stemmer, S., Mack, S. \& Allen, S. J. Two-dimensional electron gas in $\delta$-doped $\mathrm{SrTiO}_{3}$. Phys. Rev. B 82, 081103 (2010).

29. Kozuka, Y. et al. Two-dimensional normal-state quantum oscillations in a superconducting heterostructure. Nature 462, 487-490 (2009).

30. Moetakef, P. et al. Quantum oscillations from a two-dimensional electron gas at a Mott/band insulator interface. Appl. Phys. Lett. 101, 151604 (2012).

31. Harrang, J. P. et al. Quantum and classical mobility determination of the dominant scattering mechanism in the two-dimensional electron gas of an AlGaAs/GaAs heterojunction. Phys. Rev. B 32, 8126-8135 (1985).

32. Sing, M. et al. Profiling the interface electron gas of $\mathrm{LaAlO}_{3} / \mathrm{SrTiO}_{3}$ heterostructures with hard X-Ray photoelectron spectroscopy. Phys. Rev. Lett. 102, 176805 (2009).

33. Mannhart, J. \& Schlom, D. G. Semiconductor physics: the value of seeing nothing. Nature 430, 620-621 (2004).

34. Jia, C. L. \& Urban, K. Atomic-resolution measurement of oxygen concentration in oxide materials. Science 303, 2001-2004 (2004).

35. Vollmann, M., Hagenbeck, R. \& Waser, R. Grain-boundary defect chemistry of acceptor-doped titanates: inversion layer and low-field conduction. J. Am. Ceram. Soc. 80, 2301-2314 (1997).

36. Tufte, O. N. \& Chapman, P. W. Electron mobility in semiconducting strontium titanate. Phys. Rev. 155, 796-802 (1967).

37. Chen, Y. Z. \& Pryds, N. Imposed quasi-layer-by-layer homoepitaxial growth of $\mathrm{SrTiO}_{3}$ films by large area pulsed laser deposition. Thin Solid Films 519, 6330-6333 (2011).

38. Zhang, J. et al. Depth-resolved subsurface defects in chemically etched $\mathrm{SrTiO}_{3}$. Appl. Phys. Lett. 94, 092904 (2009).

39. Chambers, S. A. et al. Unintentional $\mathrm{F}$ doing of $\mathrm{SrTiO}_{3}(001)$ etched in $\mathrm{HF}$ acidstructure and electronic properties. Surface Sci. 606, 554-558 (2012).

\section{Acknowledgements}

We thank J. Fleig, F. W. Poulsen, N. Bonanos, S. Stemmer and Y.Q. Li for helpful discussions. We also thank K. Thydén, Z.I. Balogh, J.W. Andreasen, E. Johnson, Y. Zhao, X. Tang, W.W. Gao, N.Y. Wu, J. Geyti, K.V. Hansen, K. Engelbrecht and L. Theil Kuhn for their help.

\section{Author contributions}

Y.Z.C. contributed to the concept design, film growth, transport measurements, data analysis, interpretation and writing of the manuscript. N.P. and S.L. contributed to the concept design. N.B. contributed to the XPS measurements and analysis. F.T., D.V.C., N.H.A. and T.S.J. contributed to the transport measurements and analysis. F.M.Q, R.G and J.D. contributed to the SdH measurements and analysis. T.K. contributed to the STEM and EELS measurements and analysis. W.Z. contributed to the HRTEM measurements and analysis. J.R.S., A.S., J.N., L.L., B. B. and B.G.S discussed the data. All authors extensively discussed the results and the manuscript.

\section{Additional information}

Supplementary Information accompanies this paper on www.nature.com/ naturecommunications

\section{Competing financial interests: The authors declare no competing financial interests.}

Reprints and permissions information is available online at http://npg.nature.com/ reprintsandpermissions.

How to cite this article: Chen. Y. Z. et al. A high-mobility two-dimensional electron gas at the spinel/perovskite interface of $\gamma-\mathrm{Al}_{2} \mathrm{O}_{3} / \mathrm{SrTiO}_{3}$. Nat. Commun. 4:1371 doi: 10.1038/ ncomms2394 (2013). 